\title{
APROVECHAMIENTO DEL RECURSO BIOMASA A PARTIR DE LOS DESECHOS DE MADERA PARA UNA CALDERA DE VAPOR
}

\author{
EXPLOITATION OF BIOMASS RESOURCES IN \\ THE FORM OF WOOD WASTE FOR STEAM \\ BOILER
}

\author{
Juan Sebastián Arroyo-Vinueza ${ }^{1, *}$, Washington Salvatore Reina-Guzmán²
}

\section{Resumen}

Este artículo describe el potencial energético de la biomasa residual que proviene de la madera al ser utilizado en una caldera de vapor como combustible sustitutivo; esto con el fin de proporcionar una alternativa de energía limpia diferente a la actual dependencia del petróleo usado en estos dispositivos. Además, la información detalla la industria que proporciona los desechos de madera y sus diferentes formas de presentación, sus propiedades físicas y energéticas; tales como el poder calorífico, valor de acuerdo con el contenido de humedad de cada muestra de biomasa, y la equivalencia de peso de los combustibles fósiles utilizados actualmente por las calderas de vapor. Finalmente, basados en los resultados obtenidos, se realiza el análisis termodinámico que se produce en la caldera con el uso de biomasa residual, dando a conocer su balance energético, exergético y el cálculo de la entropía; lo que proporciona datos de referencia para utilizar la biomasa como una alternativa válida al uso de nuevas fuentes de energía renovables, como la biomasa residual.

Palabras clave: biomasa, caldera de vapor, combustible fósil, contaminación, madera.

\begin{abstract}
This article describes the energy potential of residual biomass from wood to be used in a steam boiler as substitute fuel; resulting in a clean energy alternative against dependence on oil use in these devices. Further, information details the industry that provides wood waste, its varying forms of presentation, their physical and energetic properties; such as: the calorific value according to the moisture content and weight equivalence of each biomass sample to replace the fossil fuels currently used by steam boilers. Finally, based on the results obtained, a thermodynamic analysis is performed on the occurrences in the boiler when used with residual biomass, giving us answers to its energy balance, exergy balance and entropy calculation; thus providing reference data to the use of residual biomass as a valid alternative in new sources of renewable energy.
\end{abstract}

Keywords: Biomass, wood, pollution, fossil fuel, steam boiler.

\footnotetext{
${ }^{1, *}$ Facultad de Ingeniería Mecánica, Universidad Politécnica Salesiana, Quito - Ecuador. Autor para correspondencia jsav187@hotmail.com

${ }^{2}$ Docente de la carrera de Ingeniería Mecánica, Universidad Politécnica Salesiana, Quito - Ecuador.
} 


\section{Introducción}

Desde el auge del petróleo y la consecuente quema de combustibles fósiles, las emisiones de gases que provocan el efecto invernadero han aumentado. Teniendo en cuenta este punto de vista, el uso de energía renovable es necesario; por ejemplo, podemos aprovechar el potencial de la energía térmica de la biomasa residual utilizando equipos tales como una caldera de vapor, que son de gran demanda en muchos procesos dentro de la industria.

Para la puesta en marcha de una caldera de vapor se utiliza regularmente como fuente de energía combustibles fósiles que contribuyen significativamente a la contaminación ambiental. Una alternativa a este proceso es utilizar biomasa residual como combustible para la caldera de vapor, pero garantizando que las condiciones de funcionamiento sean similares a las de equipos convencionales. [1]

La biomasa en nuestro medio representa una alternativa viable para el uso energético en calderas de vapor, esta aseveración se realiza considerando que existen entidades que llevan a cabo investigaciones sobre este recurso, tal es el caso de la Corporación para la Investigación Energética (CIE) y el Instituto Nacional de Eficiencia Energética y Energías Renovables (INER); un ejemplo de lo antes expuesto es el proyecto denominado El aprovechamiento energético de biomasa residual del piñón [2], que pretende utilizar este tipo de biomasa en la producción de biodiésel, para de esta manera suprimir paulatinamente el uso de derivados de petróleo en la Región Insular de nuestro país. En la misma línea de investigación la CIE ha realizado un inventario en la provincia de Pichincha de la biomasa proveniente de fuente agrícolas y animales [3], demostrando el potencial energético que posee este recurso.

Este artículo pretende entregar datos e información de la importancia de los desechos de madera, como un recurso renovable para ser utilizado como combustible en una caldera de vapor considerando las propiedades energéticas que posee.

\subsection{Biomasa residual}

La transformación de energía a partir de la biomasa representa un rendimiento idóneo neto de energía al año, y al mismo tiempo un crecimiento gradual promoviendo como un recurso sustitutivo para la generación.

No obstante, en términos de almacenamiento y transporte, la biomasa con una humedad al $50 \%$ presenta una desventaja en comparación con los combustibles derivados del petróleo; la madera presenta una baja densidad 8 GJ/t. [4]
Tabla 1. Comparation of biomass and fossil fuel energy densities [4]

\begin{tabular}{lr}
\hline Biomass (wood, $50 \%$ moisture) & $8 \mathrm{GJ} / \mathrm{t}$ \\
Liquiefed natural gas & $56 \mathrm{GJ} / \mathrm{t}$ \\
Mineral oil & $42 \mathrm{GJ} / \mathrm{t}$ \\
Coal & $28 \mathrm{GJ} / \mathrm{t}$ \\
\hline
\end{tabular}

\subsection{Tipos de madera trabajada}

Una de las principales especies que son utilizadas en las industrias, de acuerdo con el catastro facilitado por la Dirección Nacional Forestal (DNF) son eucalipto (Eucalyptus globulus), pino (Pinus radiata) y laurel (Cordia alliodora).

Tabla 2. Especies más comerciales utilizadas en la industria maderera [1]

\begin{tabular}{lc}
\hline \multicolumn{1}{c}{ Nombre científico } & $\begin{array}{c}\text { Volumen } \\
\text { movilizado }\left(\mathbf{m}^{3}\right)\end{array}$ \\
\hline Eucalipto (Eucalyptus globulus) & 87123,82 \\
Laurel (Cordia alliodora) & 63552,96 \\
Pino (Pinus radiata) & 76069,22 \\
\hline
\end{tabular}

\subsubsection{Usos y procesos de la madera}

En la provincia de Pichincha existen empresas que fabrican productos a base de biomasa, citando los más solicitados: tableros MDF, tríplex y aglomerados.

La elaboración de estos productos, que son los de mayor demanda, es a base de biomasa previamente tratada y combinada con aditivos. El tratamiento que recibe la madera una vez cortada y convertida en astillas, aserrín o virutas (Figura 1), depende de la finalidad; debe ser secada y en algunos casos lavada para eliminar hongos e impurezas, asegurando la calidad de la materia prima y el producto final. [1].

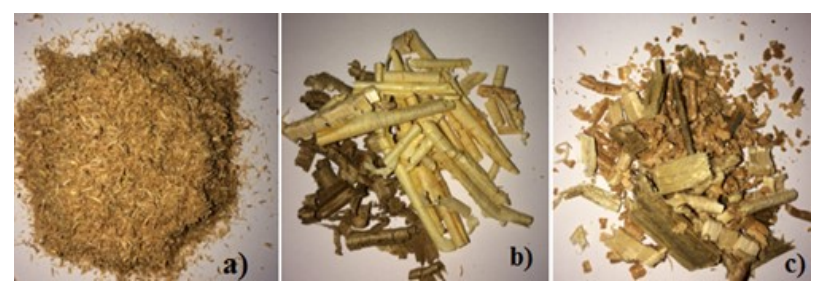

Figura 1. Formas de Biomasa: a) aserrín, b) virutas y c) astillas

\subsection{Destino de los desechos madereros, biomasa}

Los residuos obtenidos, derivan principalmente del volumen de madera trabajada, es así, que la producción puede ser estimada según la siguiente tabla. [1] 
Tabla 3. Consumo de madera por trozas de plantaciones forestales por tipo de industria y especie en Ecuador [5]

\begin{tabular}{cccc}
\hline $\begin{array}{c}\text { Tipo de } \\
\text { industria }\end{array}$ & $\begin{array}{c}\text { Consumo } \\
\left(\mathbf{1 0 0 0} \mathbf{~ m}^{\mathbf{3}}\right)\end{array}$ & $\begin{array}{c}\text { Participación } \\
\mathbf{( \% )}\end{array}$ & $\begin{array}{c}\text { Especie } \\
\text { principal }\end{array}$ \\
\hline Aserradero & 420 & 39,6 & pino \\
& Tableros & reconstruidos & \\
Aglomerados & 188 & 17,7 & pino \\
MDF & 139 & 13,1 & pino \\
Astilladora & 288 & 27,2 & eucalipto \\
Otros & 25 & 2,4 & eucalipto \\
Total & $\mathbf{1 0 6 0}$ & $\mathbf{1 0 0}$ & - \\
\hline
\end{tabular}

Cabe señalar, que el Ministerio del Ambiente a través de su Dirección Nacional Forestal regula la procedencia legal del producto forestal existente en los centros de destino final, como son los aserraderos, más no la utilización de los residuos como tal. [1]

\subsection{Almacenamiento y transporte}

La biomasa al ser un residuo, la mayoría de aserraderos tienen apilado a la intemperie, es decir, no hay un adecuado control ni manejo, no obstante, entidades como Aserradero San Alfonso, poseen un sistema centralizado de recolección mediante tubos de aspiración (Figura 2). La funcionalidad de esta técnica es de recolectar en el momento que se procesa las trozas de madera y posterior ser despachada mediante gravedad en camiones $\mathrm{u}$ otro medio de trasporte de carga.

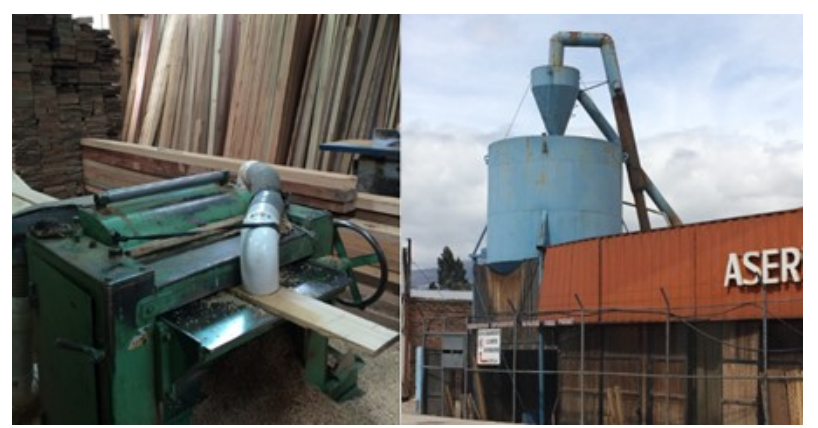

Figura 2. Recipiente vertical tipo tanque de $9 \mathrm{~m}^{3}$ para la recolección centralizada de aserrín mediante aspiración [1]

Otros aserraderos realizan la recolección de manera manual-artesanal: acumulan los residuos con una herramienta para luego entregarlos a gestores ambientales o intermediarios. De la misma manera, no existe un dato exacto de cuál es el promedio de emisión de desechos madereros, en peso o volumen, debido a que el comercio se lo realiza de un modo informal.

\section{Materiales y métodos}

\subsection{Toma de muestras de biomasa}

Las muestras de biomasa se tomaron en campo, en el Aserradero San Alfonso de la parroquia de Tumbaco, a $20 \mathrm{~km}$ al nororiente de Quito. Se recogieron $300 \mathrm{~g}$ de desechos de madera; siendo las más comerciales: pino, laurel y eucalipto.

Adicionalmente, se agregó una especie más de gran demanda como es el colorado, que también es usada en aserraderos, pero su cultivo se lleva a cabo en la zona oriental del país.

Tabla 4. Condiciones ambientales, para toma de muestras

\begin{tabular}{lc}
\hline Humedad relativa & $25 \%$ \\
Temperatura & $22{ }^{\circ} \mathrm{C}$ \\
Altitud & 2387 \\
Ubicación & La Morita, Tumbaco \\
\hline
\end{tabular}

Muestra 1: Pino (Pinus radiata) Muestra 2: Eucalipto (Eucalyptus globulus) Muestra 3: Colorado (Guarea kunthiana) Muestra 4: Laurel (Cordia alliodora)

\subsection{Propiedades fisicoquímicas y energéticas}

\subsubsection{Contenido de humedad}

La humedad representa una variable muy importante en la biomasa, pues está relacionada con el contenido de agua que posee una muestra en función de su peso. El poder calorífico que contiene este recurso es dependiente del porcentaje de humedad, en términos energéticos. Para ser determinada, se toma en cuenta en base seca o en base húmeda, siendo así $\mathrm{CH}_{\mathrm{x}}$ la humedad en base húmeda y $\mathrm{CH}_{\mathrm{u}}$ la humedad en base seca; las fórmulas para su cálculo son:

$$
\begin{aligned}
& C H_{u}(\%)=\frac{P h-P o}{P o} \\
& C H_{x}(\%)=\frac{P h-P o}{P h}
\end{aligned}
$$

Donde $\mathrm{CH}(\%)$ es el tanto por ciento de humedad, $\mathrm{Ph}$ es el peso húmedo de la muestra y Po representa el peso de la muestra después de ser secado en la estufa. [6]

El porcentaje de humedad que contiene la biomasa residual, de las cuatro muestras de madera, es un factor indispensable para el aprovechamiento energético (Figura 3). De igual manera, el contenido de humedad inferior al $50 \%$, por muestra, se toma como referencia en base seca; por lo tanto, los resultados obtenidos son idóneos para su uso energético. 


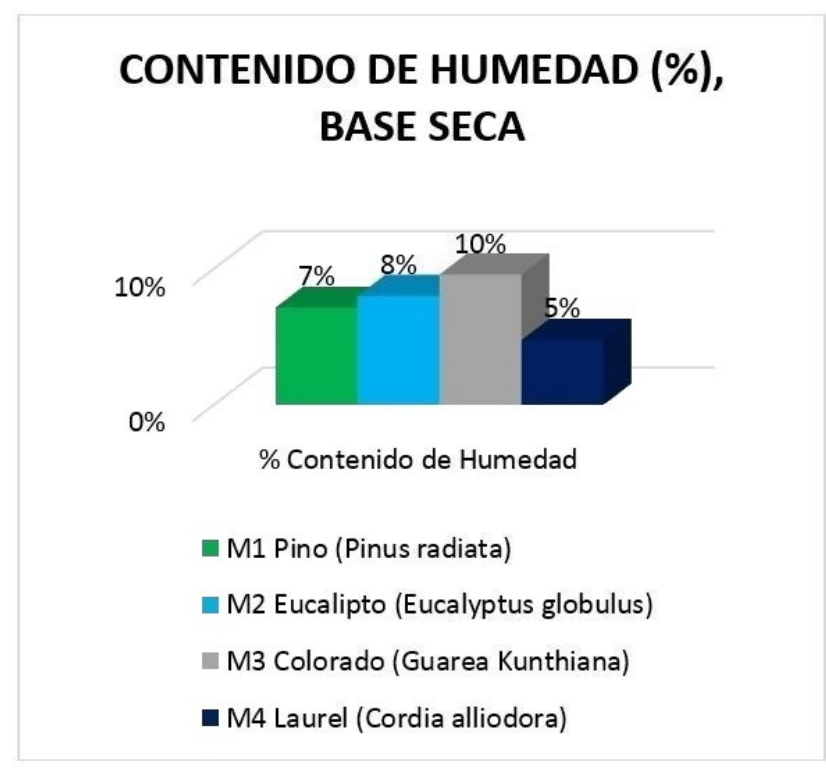

Figura 3. Porcentaje de humedad obtenidos por muestra [1]

\subsubsection{Densidad aparente}

Esta propiedad física que poseen los biocombustibles sólidos también es dependiente del contenido de humedad [7], cuanto menos contenido de agua menor es su densidad, pero se incrementa su poder calorífico y densidad energética.

Tabla 5. Condiciones ambientales, para toma de muestras

\begin{tabular}{lc}
\hline \multicolumn{1}{c}{ Muestra / Especie } & $\begin{array}{c}\text { Densidad } \\
\text { aparente } \\
\text { g/ml }\end{array}$ \\
\hline Muestra 1: Pino (Pinus patula) & 0,048 \\
Muestra 2: Eucalipto (Eucalyptus globulus) & 0,102 \\
Muestra 3: Colorado (Guarea kunthiana) & 0,161 \\
Muestra 4: Laurel (Cordia alliodora) & 0,66 \\
\hline
\end{tabular}

Para aumentar la densidad aparente existen procesos mecánicos como la elaboración de pellets, compresión de aserrín mezclado con aditivos químicos, elevando el rendimiento de la caldera puesto que habría una mejor combustión y menos contenido de cenizas; aunque se incrementaría costos en su fabricación.

\subsubsection{Composición elemental de la madera}

La madera en sus diferentes especies posee una composición idéntica, al igual que en sus distintas partes como son tronco y ramas de un mismo árbol (Figura 4), [8].

A diferencia de los combustibles fósiles que contienen una gran proporción de carbono 84-86 \% [1], la biomasa proveniente de la madera lleva ventaja. Adicionalmente, la biomasa al ser combustionada, los gases resultantes $\mathrm{CO}$ y $\mathrm{CO}_{2}$ son captados nuevamente por las plantas por medio de su proceso natural fotosíntesis.

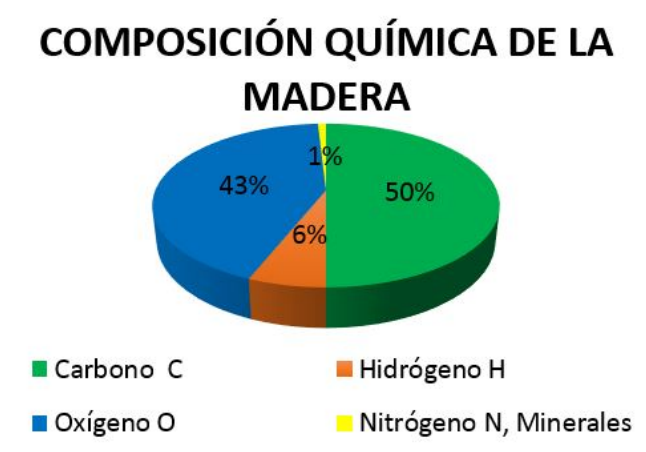

Figura 4. Composición química de la madera [8]

\subsubsection{Contenido de cenizas}

Para especies como el pino y el eucalipto se tiene un porcentaje de cenizas inferior al $0,5 \%$, lo cual refleja que al combustionar estas maderas poseen menos materia sólida no combustionada.

Tabla 6. Contenido de cenizas por muestra [1]

\begin{tabular}{lc}
\hline Muestra / Especie & $\begin{array}{c}\text { \% de muestra } \\
\text { /especie } \\
\text { cenizas }\end{array}$ \\
\hline Muestra 1: Pino (Pinus patula) & 0,3169 \\
Muestra 2: Eucalipto (Eucalyptus globulus) & 0,3724 \\
Muestra 3: Colorado (Guarea kunthiana) & 1,2061 \\
Muestra 4: Laurel (Cordia alliodora) & 1,5375 \\
\hline
\end{tabular}

Las cenizas, producto de la combustión de la madera, por su alto contenido de carbonato de potasio o potasa, son usadas como fertilizante para cultivos.

\subsubsection{Poder calorífico}

El poder calorífico se define como la cantidad de energía liberada en forma de calor en un kilogramo, o en un metro cúbico de combustible cuando se quema por completo en un ambiente a presión constante de $101 \mathrm{Kpa}$ y $25^{\circ} \mathrm{C}$, en condiciones normales. En otros términos, el poder calorífico es el valor absoluto de la entalpía.

$$
\text { Podercalorífico }=\left|h_{e}\right|[k J / k g](\text { combustible })
$$

Poder calorífico superior PCS o HHV por sus siglas en inglés, es aquel desprendido al final de la combustión, cuando el agua del producto se encuentra en forma líquida.

Poder calorífico inferior PCI o LHV por sus siglas en inglés, cuando el $\mathrm{H}_{2} \mathrm{O}$ en los productos está en forma de vapor. 
Para determinar el contenido calorífico superior o bruto se empleó un calorímetro adiabático. Bajo la norma ASTM D-240 que recomienda el fabricante se ejecuta este ensayo por cada muestra de biomasa.

Tabla 7. Poder calorífico superior e inferior [1]

\begin{tabular}{lcc}
\hline \multicolumn{1}{c}{ Muestra / Especie } & $\begin{array}{c}\text { Poder } \\
\text { calorífico } \\
\text { superior } \\
\mathbf{M J} / \mathbf{k g}\end{array}$ & $\begin{array}{c}\text { Poder } \\
\text { calorífico } \\
\text { inferior } \\
\mathbf{M J} / \mathbf{k g}\end{array}$ \\
\hline Muestra 1: Pino (Pinus patula) & 17,7807 & 14,7597 \\
Muestra 2: Eucalipto (Eucalyptus globulus) & 17,6244 & 14,5028 \\
Muestra 3: Colorado (Guarea kunthiana) & 18,627 & 14,9973 \\
Muestra 4: Laurel (Cordia alliodora) & 18,913 & 16,2133 \\
\hline
\end{tabular}

Es de gran importancia acotar que en todos los procesos técnicos industriales [8] son de interés solo el poder calorífico inferior PCI, esto debido a que el calor de la condensación del vapor de agua que contienen los gases resultantes de la combustión no resulta utilizable.

$$
P C I=\frac{P C S-600(u+9 h)}{1+u}
$$

Donde, $P C S$ es el poder calorífico superior obtenido mediante el uso de un calorímetro adiabático, 600 es el calor de vaporización a $0{ }^{\circ} \mathrm{C}[\mathrm{kcal} / \mathrm{kg}], u$ representa la humedad del combustible (referida al peso seco), 9 kilos de agua que se forman al oxidar un kilo de hidrógeno y $h$ la proporción de hidrógeno 6,1 \%.

Esta ecuación nos permite obtener el grado calorífico neto de una sustancia sólida conociendo su humedad en base seca y su poder calorífico superior o bruto (Figura 5) que se obtiene en laboratorio mediante un calorímetro como se detalla en la Tabla 7.

Hay que tomar mucha atención en los porcentajes de humedad, pues de este resultado depende el grado calorífico que se puede aprovechar. Procesos a gran escala como el secado, previo a la combustión en la caldera u otro dispositivo, son de vital importancia; es ahí donde desempeña un papel importante la humedad de la biomasa.

A mayor humedad se obtiene menos grado calorífico (Figura 6), por lo que es importante conocer el origen y la manipulación que se ha dado a la biomasa residual.

\subsection{Datos técnicos de la caldera}

El generador de vapor a analizar es utilizado en la microindustria alimenticia. La caldera seleccionada es pirotubular marca Distral, de fabricación colombiana [9].

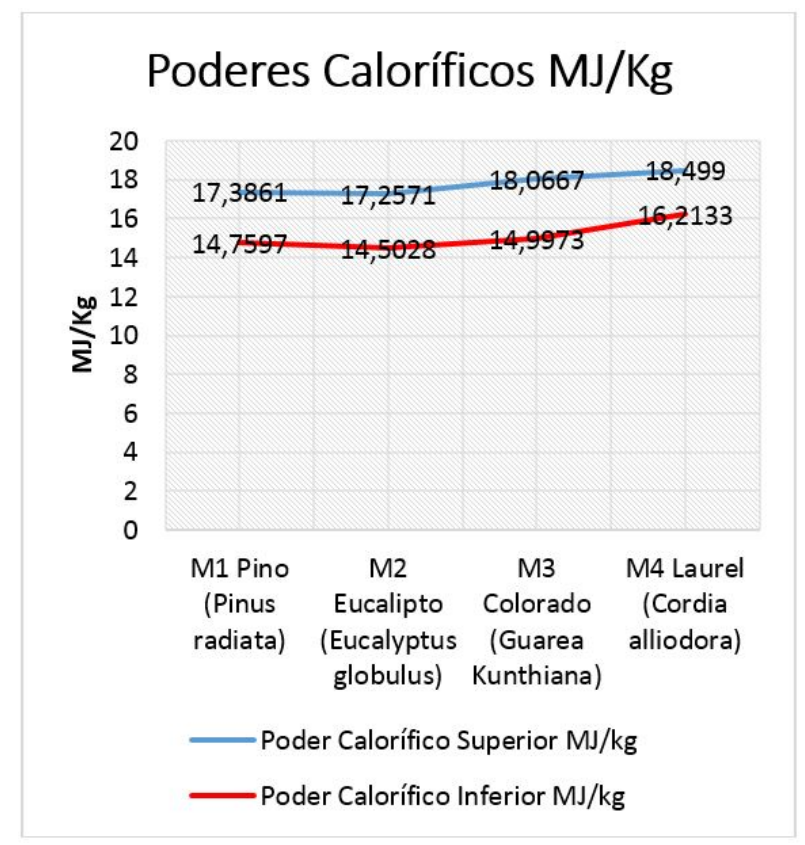

Figura 5. Comparación de poderes caloríficos obtenidos [1]

Se seleccionó este caldero debido a que, para el análisis, se requiere datos experimentales tomados en campo; adicional en el país no existen calderos que funcionan con combustibles de biomasa proveniente de madera.

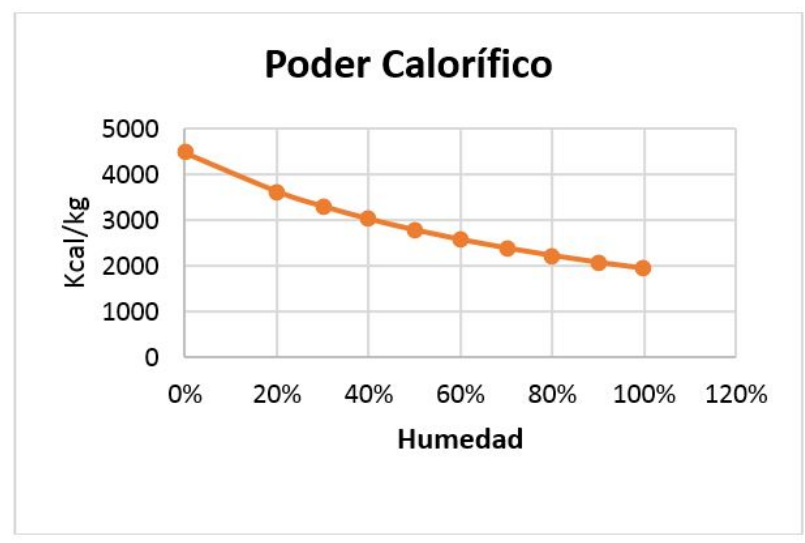

Figura 6. Relación poder calorífico con porcentaje de humedad [1]

Tabla 8. Datos técnicos de la caldera seleccionada [9]

\begin{tabular}{ll}
\hline BHP & $30 \mathrm{HP}$ \\
Tiempo de trabajo & $8 \mathrm{~h} / \mathrm{día}$ \\
Consumo de combustible fósil & $5 \mathrm{litros} / \mathrm{h}$ \\
* Consumo de combustible biomasa & $12,45 \mathrm{~kg} / \mathrm{h}$ \\
tipo & diésel 2 \\
PCI Diésel 2 kcal/1 & 8791,38 \\
\% retorno condensado & $89,1 \%$ aprox. \\
Presión de trabajo & 50 PSI \\
\hline *Valores infieren de acuerdo con el PCI, ver Tabla 7.
\end{tabular}




\subsection{Flujo de biomasa equivalente por muestra}

Tabla 9. Datos técnicos de la caldera seleccionada [9]

\begin{tabular}{lcc}
\hline \multicolumn{1}{c}{ Tipo de Biomasa } & $\begin{array}{c}\text { PCI } \\
\text { (kcal/kg) }\end{array}$ & $\begin{array}{c}\text { Equivalencia en } \\
\text { flujo de biomasa } \\
\text { para caldera } \\
\mathbf{( k g / h )}\end{array}$ \\
\hline Muestra 1: Pino (Pinus radiata) & 3531,02 & 12,45 \\
Muestra 2: Eucalipto (Eucalyptus globulus) & 3469,58 & 12,67 \\
Muestra 3: Colorado (Guarea kunthiana) & 3587,87 & 12,25 \\
Muestra 4: Laurel (Cordia alliodora) & 3878,78 & 11,33 \\
\hline
\end{tabular}

En la Tabla 9 se hace mención de la cantidad de biomasa necesaria para poder suplir al combustible fósil que es usado en esta caldera, previamente conociendo el poder calorífico neto. Así para la especie de biomasa (Pinus radiata) se requiere de $12,45 \mathrm{~kg} / \mathrm{h}$ de este, en reemplazo de los 5 galones de diésel por hora.

\subsection{Ecuaciones de gobierno para una caldera de vapor}

\subsubsection{Balance de energía}

El balance energético de un sistema hace referencia a la primera ley de la termodinámica donde determina que la energía se transforma, no se puede destruir ni crear durante un proceso.

$$
\begin{gathered}
\text { (E. entrada })-(\text { E. salida })=(\text { E. sistema }) \\
Q=\Delta E+W
\end{gathered}
$$

Donde, $Q$ es el calor generado por el dispositivo, $\Delta \mathrm{E}$ la energía del sistema y $W$ es el trabajo realizado. Para obtener el balance térmico energético de una caldera, hay que considerar dos aspectos: la energía proveniente del combustible y las pérdidas generadas durante la oxidación del mismo.

Los generadores de vapor o calderas se consideran sistemas estacionarios puesto que sus energías potencial y cinética son despreciables, por consiguiente, la energía total de un sistema es igual al cambio de su energía interna.

$$
Q-W=\Delta U
$$

En calderos, dispositivos a volumen constante, no generan trabajo [10]:

$$
\begin{gathered}
Q=\Delta U \\
\dot{Q}=\dot{m} \times P C I
\end{gathered}
$$

Donde $\dot{m}$ es el flujo de combustible equivalente de biomasa (ver Tabla 9) y PCI corresponde al poder calorífico inferior obtenido de la ecuación (3).

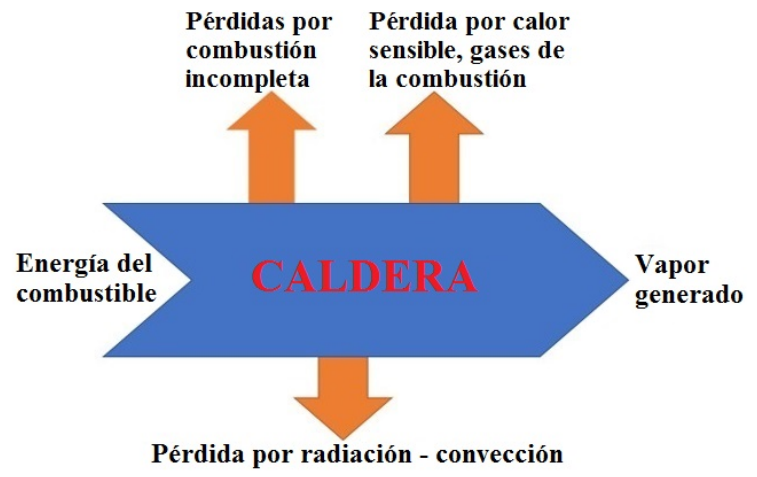

Figura 7. Diagrama de sankey de una caldera [1]

\subsubsection{Balance energético caldera}

$$
\dot{Q}_{\text {entrada }}=\dot{Q}_{\text {salida }}
$$

$$
\dot{Q}_{\text {salida }}=\dot{Q}_{\text {entrada }}-\sum \dot{Q}_{\text {pérdidas }}
$$

Donde $\dot{Q}_{\text {entrada }}$ es la cantidad de calor entrante de combustible, $\dot{Q}_{\text {salida }}$ cantidad de calor de salida y $\sum \dot{Q}_{\text {pérdidas }}$ referido a la sumatoria, cantidad de calor total perdido.

El porcentaje de pérdidas por el calor sensible de los gases que se suscita en el conjunto hornocaldera: radiación-convección, por purgas existentes, combustión incompleta; considera el $23 \%$ de pérdidas del total de energía entrante de combustible (Figura 7) [11,12].

$$
\sum \dot{Q}_{\text {pérdidas }}=0,23 \times \dot{Q}_{\text {entrada }}
$$

Tabla 10. Balance energético obtenido por cada muestra [1]

\begin{tabular}{lccc}
\hline Tipo de muestra & $\begin{array}{c}\text { Q entrada } \\
\mathbf{k c a l} / \mathbf{h}\end{array}$ & $\begin{array}{c}\text { Q salida } \\
\mathbf{k c a l} / \mathbf{h}\end{array}$ & $\begin{array}{c}\text { Q pérdidas } \\
\mathbf{0 , 2 3} \mathbf{\%}\end{array}$ \\
\hline $\begin{array}{l}\text { Muestra1: Pino } \\
\text { (Pinus radiata) }\end{array}$ & 43961,2 & 33850,124 & 10111,07 \\
$\begin{array}{l}\text { Muestra 2: Eucalipto } \\
\text { (Eucalyptus globulus) }\end{array}$ & 43959,57 & 33848,51 & 10110,7 \\
$\begin{array}{l}\text { Muestra 3: Colorado } \\
\text { (Guarea kunthiana) }\end{array}$ & 43951,41 & 33840,34 & 10108,24 \\
$\begin{array}{l}\text { Muestra 4: Laurel } \\
\text { (Cordia alliodora) }\end{array}$ & 43946,57 & 33835,51 & 10107,71 \\
Rendimiento \% & & $0,77 \rightarrow 77 \%$ & \\
\hline
\end{tabular}

Cabe recalcar que, cuantas menos pérdidas se obtenga la combustión será más completa y por consiguiente el rendimiento aumentará, de ahí la importancia del estudio de eficiencia energética de los dispositivos termodinámicos. 


\subsubsection{Balance de exergía o disponibilidad}

La primera ley de la termodinámica menciona que la energía no se crea ni se destruye, solo se transforma, por lo tanto, la exergía es la propiedad que determina el potencial de aprovechamiento de dicha energía para producir un trabajo; es decir, el primer principio trata de la cantidad de energía mientras la exergía trata la calidad de esta energía.

Para dispositivos como calderas, los cuales no generan trabajo, el cálculo de la eficiencia exergética o eficiencia de la segunda ley hace referencia a lograr el objetivo del proceso en relación con los insumos para el proceso en términos de cambio de exergía o transferencias [13]. El balance de exergía parte de la ecuación para un volumen de control:

$$
\begin{aligned}
\Psi_{2}-\Psi_{1}=\left(h_{2}-h_{1}\right)- & T_{0}\left(S_{2}-S_{1}\right) \\
& +\frac{C_{2}^{2}-C_{1}^{2}}{2}+g\left(z_{2}-z_{1}\right)
\end{aligned}
$$

Las energías cinética y potencial son despreciables para la caldera:

$$
\begin{gathered}
\Psi_{2}-\Psi_{1}=\left(h_{2}-h_{1}\right)-T_{0}\left(S_{2}-S_{1}\right) \\
\Psi_{2}-\Psi_{1}=(2492,45-579,56) \\
-293 K(6,3707-1,72174) \\
\Psi_{2}-\Psi_{1}=550,74 \frac{\mathrm{kJ}}{\mathrm{kgH} O}
\end{gathered}
$$

Donde $\Psi_{2}-\Psi_{1}$ es el aumento de exergía del agua por kilogramos de agua, $\left(h_{2}-h_{1}\right) ;\left(S_{2}-S_{1}\right)$ es la diferencia de entalpía y entropía respectivamente y $T_{0}$ es la temperatura ambiente $20{ }^{\circ} \mathrm{C}$.

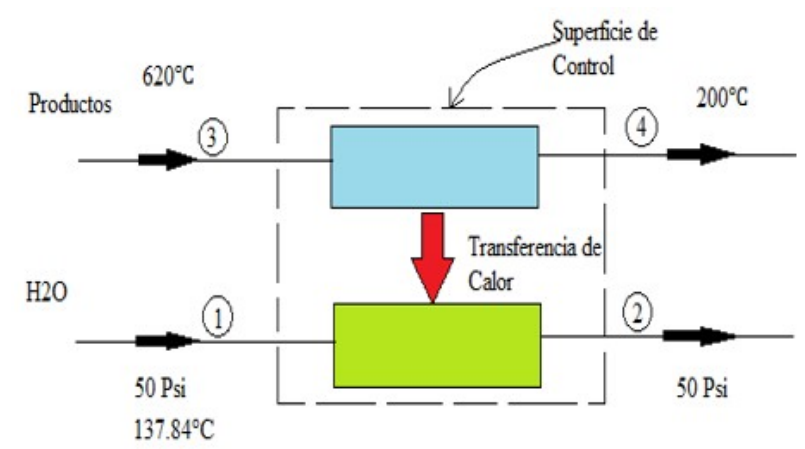

Figura 8. Volumen de control para caldera [13]

Para el volumen de control las ecuaciones que rigen (Figura 8) [13]:

Ecuación de la continuidad:

$$
\left(\dot{m}_{1}\right)_{\mathrm{H}_{2} \mathrm{O}}=\left(\dot{m}_{2}\right)_{\mathrm{H}_{2} \mathrm{O}}
$$

$$
\left(\dot{m}_{3}\right)_{\text {productos }}=\left(\dot{m}_{4}\right)_{\text {productos }}
$$

De la Primera ley, se obtiene:

$$
\begin{aligned}
\left(\dot{m}_{1} h_{1}\right)_{\mathrm{H}_{2} \mathrm{O}}+ & \left(\dot{m}_{3} h_{3}\right)_{\text {productos }}= \\
& \left(\dot{m}_{2} h_{2}\right)_{\mathrm{H}_{2} \mathrm{O}}+\left(\dot{m}_{4} h_{4}\right)_{\text {productos }}
\end{aligned}
$$

Para la relación de flujo de los productos y el flujo másico de agua, se toma en cuenta las ecuaciones (11), (12) y (13).

$$
\begin{gathered}
\frac{\dot{m}_{\text {productos }}}{\dot{m}_{\mathrm{H}_{2} \mathrm{O}}}=\frac{\left(h_{2}-h_{1}\right)_{\mathrm{H}_{2} \mathrm{O}}}{\left(h_{3}-h_{4}\right)_{\text {productos }}} \\
\frac{\dot{m}_{\text {productos }}}{\dot{m}_{\mathrm{H}_{2} \mathrm{O}}}=\frac{2492.45-579,56}{1,87(620-200)} \\
\frac{\dot{m}_{\text {productos }}}{\dot{m}_{\mathrm{H}_{2} \mathrm{O}}}=2,435
\end{gathered}
$$

El cambio de entropía para los productos (gas ideal), siendo a presión constante es:

$$
\left(s_{4}-s_{3}\right)_{\text {productos }}=C_{p} \times \ln \frac{T_{e}}{T_{i}}
$$

La disminución de exergía de los productos se obtiene de la ecuación (10):

$$
\begin{array}{r}
\frac{\dot{m}_{\text {productos }}}{\dot{m}_{\mathrm{H}_{2} \mathrm{O}}}\left(\Psi_{3}-\Psi_{4}\right)=\frac{\dot{m}_{\text {productos }}}{\dot{m}_{\mathrm{H}_{2} \mathrm{O}}}\left(h_{3}-h_{4}\right) \\
-T_{0}\left(s_{4}-s_{3}\right)
\end{array}
$$

Reemplazando la ecuación (15) en (16), se obtiene:

$$
\begin{aligned}
& \frac{\dot{m}_{\text {productos }}}{\dot{m}_{\mathrm{H}_{2} \mathrm{O}}}\left(\Psi_{3}-\Psi_{4}\right)= \\
& \frac{\dot{m}_{\text {productos }}}{\dot{m}_{\mathrm{H}_{2} \mathrm{O}}}\left[\left(h_{3}-h_{4}\right)-T_{0}\left(C_{p} \times \ln \frac{T_{3}}{T_{4}}\right)\right]
\end{aligned}
$$

Sustituyendo valores:

$$
\begin{gathered}
\frac{\dot{m}_{\text {productos }}}{\dot{m}_{\mathrm{H}_{2} \mathrm{O}}}\left(\Psi_{3}-\Psi_{4}\right)= \\
2,435\left[1,87(620-200)-293\left(1,87 \ln \frac{893}{473}\right)\right] \\
\Psi_{3}-\Psi_{4}=1064,60 \frac{\mathrm{kJ}}{\mathrm{kg} \mathrm{H} \mathrm{H}_{2} \mathrm{O}}
\end{gathered}
$$

La eficiencia exergética o de la segunda ley en función del balance:

$$
\begin{gathered}
\eta_{\text {exergético }}=\frac{\dot{m}_{\mathrm{H}_{2} \mathrm{O}}\left(\Psi_{2}-\Psi_{1}\right)}{\dot{m}_{\text {productos }}\left(\Psi_{3}-\Psi_{4}\right)} \\
\eta_{\text {exergético }}=\frac{550,74}{1064,60} \\
\eta_{\text {exergético }}=0,5173 \rightarrow 51,7 \%
\end{gathered}
$$




\subsubsection{Entropía}

La entropía es una propiedad que no se conserva, por lo tanto, no existe el principio de la conservación de energía. Esta energía se conserva solamente durante un proceso reversible, realizado y se incrementa durante todos los procesos reales. La entropía generada o creada se presenta por la presencia de las irreversibilidades del sistema. La entropía generada durante un proceso se llama generación de entropía y su valor depende del proceso, por lo que no es una propiedad del sistema.

$$
\Delta_{S \text { del sistema }}=s_{2}-s_{1}
$$

$$
\Delta_{S \text { productos }}=s_{4}-s_{3}
$$

$$
\Delta_{S \text { Total }}=\Delta_{S \text { productos }}+\Delta_{S \text { del sistema }}
$$

Para el cálculo correspondiente se debe conocer los siguientes datos:

$$
\begin{gathered}
P_{\text {salida vapor }}=50 P S I=344,74 \mathrm{KPa} \\
X_{\text {calidad vapor }}=0,89 \\
{ }^{\circ} \mathrm{T}_{\mathrm{H}_{2} \mathrm{O} \text { entrada caldera }}=46,5^{\circ} \mathrm{C} \\
{ }^{\circ} \mathrm{T}_{3}=620^{\circ} \mathrm{C}+273=893 \mathrm{~K} \\
{ }^{\circ} \mathrm{T}_{3}=200^{\circ} \mathrm{C}+273=473 \mathrm{~K}
\end{gathered}
$$

Valores obtenidos interpolando [10]:

$$
\begin{gathered}
{ }^{\circ} T_{\text {sat }}=137,84^{\circ} \mathrm{C} \\
s_{f}=1,72174 \frac{\mathrm{kJ}}{\mathrm{kgK}} \quad s_{g}=6,9454 \frac{\mathrm{kJ}}{\mathrm{kgK}} \\
s_{f g}=5,22367 \frac{\mathrm{kJ}}{\mathrm{kgK}} \\
h_{f}=h_{1}=579,56 \frac{\mathrm{kJ}}{\mathrm{kg}} \quad h_{f g}=579,56 \frac{\mathrm{kJ}}{\mathrm{kg}}
\end{gathered}
$$

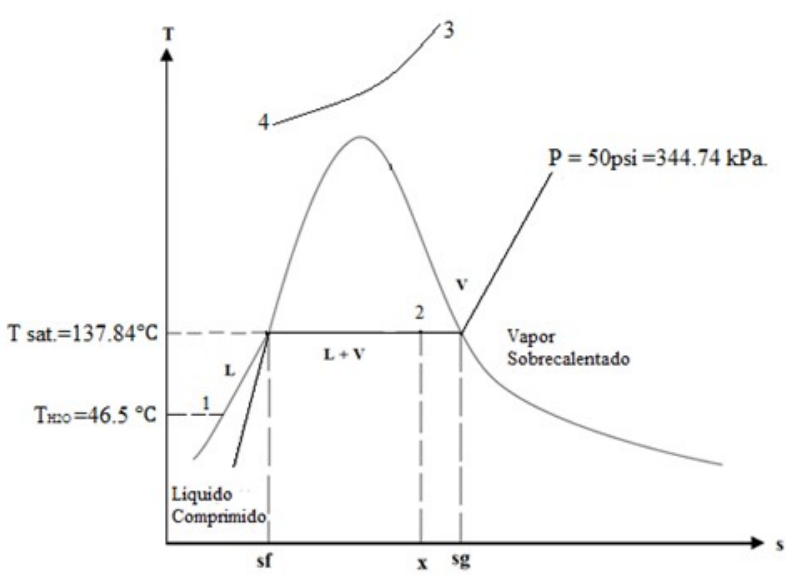

Figura 9. Diagrama temperatura-entropía de caldero pirotubular [1]

$$
\begin{gathered}
\Delta S=S_{2}-S_{1} \\
s_{1}=s_{f}=1,72174 \frac{\mathrm{kJ}}{\mathrm{kgK}} \\
s_{2}=s_{f}+x s_{f g} \\
s_{2}=1,72174+(0,89 \times 5,22367) \\
s_{2}=6,3707 \frac{\mathrm{kJ}}{\mathrm{kgK}} \\
\Delta S=(6,3707-1,72174) \\
\Delta S=4,6490 \frac{\mathrm{kJ}}{\mathrm{kgK}}
\end{gathered}
$$

Para el cálculo de la variación de entropía de los productos en la caldera, como referencia, se parte de las ecuaciones (14) y (15), obteniendo el cambio de entropía de los productos:

$$
\frac{\dot{m}_{\text {productos }}}{\dot{m}_{\mathrm{H}_{2} \mathrm{O}}}\left(s_{4}-s_{3}\right)=\frac{\dot{m}_{\text {productos }}}{\dot{m}_{\mathrm{H}_{2} \mathrm{O}}}-C_{p} \ln \left(\frac{T_{e}}{T_{i}}\right)
$$

Para el cálculo de la variación de entropía de los productos en la caldera, se sitúa en los puntos 3 y 4 del diagrama $\mathrm{T} \mathrm{f}(\mathrm{s})$ de la figura 9:

$$
\begin{gathered}
\Delta_{\text {s productos }}=\left(S_{4}-S_{3}\right)_{\text {productos }}=C_{p} \times \ln \left(\frac{T_{e}}{T_{i}}\right) \\
\frac{\dot{m}_{\text {productos }}}{\dot{m}_{H_{2} O}}\left(s_{4}-s_{3}\right)=\frac{\dot{m}_{\text {productos }}}{\dot{m}_{\mathrm{H}_{2} \mathrm{O}}}-C_{p} \times \ln \left(\frac{T_{e}}{T_{i}}\right) \\
\left(S_{4}-S_{3}\right)_{\text {productos }}=2,435 \times(-1,87) \ln \left(\frac{893}{473}\right)
\end{gathered}
$$




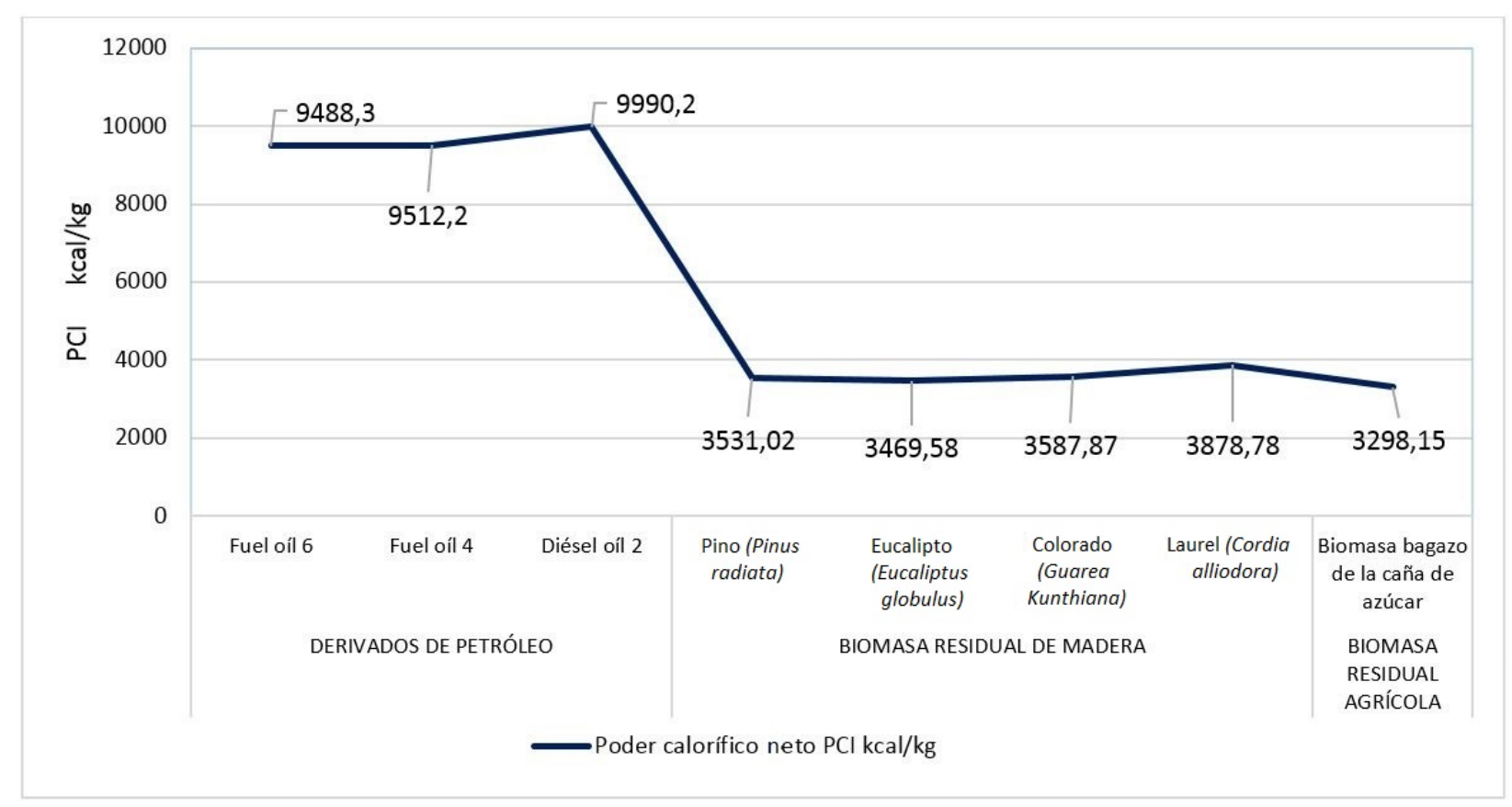

Figura 10. Comparación de poderes caloríficos netos entre combustibles fósiles y biomasa residual

$$
\left(S_{4}-S_{3}\right)_{\text {productos }}==-2,8937 \frac{\mathrm{kJ}}{\mathrm{kg} \mathrm{K}}
$$

$\Delta_{s \text { Total }}=\Delta_{S \text { productos }}+\Delta_{S \text { del sistema }}$

$$
\begin{gathered}
\Delta_{s \text { Total }}=-2,8937+4,6490 \frac{\mathrm{kJ}}{\mathrm{kgK}} \\
\Delta_{\text {s Total }}=1,7553 \frac{\mathrm{kJ}}{\mathrm{kgK}}
\end{gathered}
$$

\section{$\Delta_{s \text { Total }}>0 \therefore$ Proceso irrever sible}

Se menciona que la entropía de un sistema puede ser negativa, pero la generación total de entropía no, por lo que el resultado obtenido cumple con el enunciado [10].

\section{Resultados}

El porcentaje de humedad de las muestras seleccionadas oscila entre el 7 y $10 \%$, al mismo tiempo, el poder calorífico neto obtenido frente a los combustibles derivados del petróleo utilizados en caderas de vapor, llevan una ventaja de 3 a 1 como se muestra en la Figura 10; sin embargo, emiten gases contaminantes por su alto contenido de carbón fósil.

Equipos como la caldera de vapor el porcentaje de pérdidas corresponde al $23 \%$, donde una parte corresponde a los residuos de cenizas, para especies como el laurel y colorado son altas entre el 1,2 y $1,5 \%$; esto puede ser ocasionado por el suministro inadecuado de oxígeno al ocurrir la combustión.

La combustión directa de biomasa es el método más idóneo y económico, debido a que en el diseño no representa muchas complejidades.

\section{Conclusiones}

La biomasa producida por los desechos de la madera en aserraderos es un potencial idóneo para la producción de energía térmica, aplicada en distintas maneras como, por ejemplo, generadores de vapor, hornos, turbinas, etc.

Los poderes caloríficos de cada tipo de biomasa existente en los aserraderos, depende del almacenamiento que se da, puesto que de ahí deriva la cantidad de energía en función de la humedad. De preferencia la biomasa con un $10 \%$ de humedad es óptima para su aprovechamiento energético.

Es recomendable utilizar biomasa residual de madera con un grado del $10 \%$ en base seca máximo de humedad para dispositivo como la caldera, evitando así la corrosión de las partes constitutivas

La calidad de la energía o exergía medida en la caldera es del $51 \%$, siendo el $49 \%$ del total de vapor generado un desperdicio, esto debido a las irreversibilidades que ocurre al interior del dispositivo. 


\section{Referencias}

[1] J. S. Arroyo, "Aprovechamiento del recurso biomasa a partir de los desechos de madera para una caldera de vapor," Proyecto de Titulación, Universidad Politécnica Salesiana, Quito, Ecuador, 2015.

[2] Instituto Nacional de Eficiencia Energética y Energías Renovables (INER), "Biomasa," Quito, Ecuador, enero, 2013.

[3] Corporación para la Investigación Energética (CIE), "Inventario de biomasa residual de la poscosecha y agroindustria a nivel cantonal y provincial," Quito, Pichincha, Ecuador, 2013.

[4] P. McKendry, "Energy production from biomass (part 2): conversion technologies," Bioresource Technology, pp. 52-53, 2001.

[5] Ecuador Forestal, "Planificación estratégica, transformación y comercialización de madera en el Ecuador," Quito, 2007.

[6] FAO. (2004) Terminología unificada de bioenergía. [Online]. Available: http://www.fao.org/docrep/ 009/j6439s/j6439s00.htm. (2004).
[7] G. San Miguel and F. Gutiérrez, Tecnologías para el uso y transformación de biomasa energética. Ediciones Mundi-Prensa, Madrid, 2015.

[8] F. Kollman, "Tecnología de la madera," Gráficas Reunidas, pp. 137-262, Múnich, 1951.

[9] J. Gallardo, "Balance de masa y energía de un caldero marca distral de una microindustria de alimentos," Proyecto de Titulación, Universidad Central del Ecuador, Quito, Ecuador, 2000.

[10] Y. Cengel and M. Boles, Termodinámica. Mc Graw-Hill, México D.F., 2009.

[11] M. Echeverría and O. López, "Caracterización energética de la cascarilla de arroz para su aplicación en la generación de energía termoeléctrica," Proyecto de Titulación, Escuela Politécnica Nacional, Quito, Ecuador, 2010.

[12] A. Aguinaga, "Seminarios industriales de generación de vapor," Escuela Politécnica Nacional, Quito, Ecuador, 2010.

[13] G. Van Wylen, "Fundamento de termodinámica," Limusa S. A., México, 2003. 